\title{
Estudio de la diversidad taxonómica y funcional de los macroinvertebrados en un río de montaña Andino
}

\author{
Camila Andrea Díaz Rojas ${ }^{1}$, Ángela Judith Motta-Díaz ${ }^{1 *} \&$ Nelson Aranguren-Riaño ${ }^{1}$ \\ 1. Unidad de Ecología en Sistemas Acuáticos - UDESA, Universidad Pedagógica y Tecnológica de Colombia; \\ camila.diaz@uptc.edu.co, angela.motta@uptc.edu.co,nelson.aranguren@uptc.edu.co \\ * Correspondencia
}

Recibido 18-X-2019. Corregido 07-VII-2020. Aceptado 31-VIII-2020.

\begin{abstract}
Study of the taxonomic and functional diversity of the macroinvertebrate assemblages in an Andean mountain river. Introduction: The structure, taxonomic diversity and functional diversity of the aquatic macroinvertebrate assemblages are estimators sensitive to variations in the surrounding environment. The physical environment is a key factor, where the substrate and hydraulic dynamics modify the availability of resources, the structuring, the functioning of the community and the ecosystem. Objective: Determine the structure, taxonomic diversity and functional diversity of the macroinvertebrate assemblages under different abiotic conditions in the Hato Laguna river, a tributary of Lake Tota. Methods: 120 biological samples were collected in four sampling events in two reaches in the river. Depth, flow velocity, channel width and roughness of the substrate were measured in situ. The functional traits evaluated were: body length, body shape, type of breathing, type of locomotion, trophic guild and functional feeding groups. Results: A total of 38 taxa were identified; Diptera, Coleoptera and Trichoptera orders presented the highest abundance of families and genera. Orthocladiinae, Andesiops sp. and Simulium sp. were more abundant groups under high-velocity conditions in the upper reaches. Nectopsyche sp. was abundant in the lower sections related to lower velocity and greater channel width. According to the taxonomic diversity indices, variation by reaches and samplings was presented without observing trends. In the upper reaches, the MOPF trophic guild (collectors), in tegument respiration, and small body size were characteristic. In the lower reaches, the assembly was of the scraper type with gill breathing and flattened body shape. The richness, divergence and functional evenness were higher in the upper reaches. In the Canonical Correspondence Analysis, the highest values of width and speed were related to categories attached to the substrate and endobenthic, while low width and roughness were related to the categories of flattened body shape, microphytes food resource, and maximum body size. Conclusions: The system presented a variation in the abiotic conditions, in the structure, taxonomic and functional diversity of the macroinvertebrates. The role of the flow velocity as a determining variable of the structure and function of the community is highlighted. Likewise, functional diversity stands out as an expression of the described traits, which is why it is related in the upper reaches with the dominance of taxa such as Orthocladiinae, Chironominae, Simulium sp. and Andesiops sp.
\end{abstract}

Key words: aquatic insects; functional features; physical variables; hydraulics; Tota Lake.

Díaz Rojas, C.A., Motta Díaz, A.J., \& Aranguren Riaño, N.J. (2020). Estudio de la diversidad taxonómica y funcional de los macroinvertebrados en un río de montaña Andino. Revista de Biología Tropical, 68(S2), S132-S149.

Los ecosistemas lóticos de montaña son sistemas complejos en su estructura y funcionamiento; en estos ocurren diferentes procesos que involucran variables físicas, químicas y biológicas, que interactúan de forma sinérgica y hacen que estos sistemas tengan alta heterogeneidad espacio-temporal (Allan \& Castillo, 2007; Elosegi \& Sabater, 2009). Así, esta variación espacio-temporal contribuye a la dinámica y distribución de las comunidades biológicas 
(Brooks, Haeusler, Reinfelds, \& Williams, 2005; Vimos-Lojano, Martínez-Capel, Hampel, \& Vázquez, 2019). Particularmente, los arroyos y ríos de los Andes albergan una alta diversidad biológica que se integra en los procesos ecosistémicos y determina el funcionamiento de éstos. Sin embargo, la más básica información sobre esta diversidad, historias de vida y roles ecológicos, es escasa para varios ecosistemas andinos de Colombia, Ecuador, Bolivia y Perú (Ramírez \& Gutiérrez-Fonseca, 2014; Encalada, Flecker, LeRoy, Suárez, Herrera, RíosTouma, Jumani, Larson \& Anderson, 2019).

El ensamblaje de macroinvertebrados acuáticos, es uno de los más representativos en los ríos de montaña tropical, se caracteriza por tener una fuerte relación con el ambiente circundante, por tanto, sus poblaciones son sensibles a las variaciones de las condiciones ambientales locales y regionales (Merritt, Cummins \& Berg, 2008). Además, estos organismos contribuyen a la estructuración y funcionamiento del ecosistema, por ejemplo, mediante el procesamiento de materia orgánica y los flujos de nutrientes dentro de la red trófica (Bonada, Prat, Resh, \& Statzner, 2006; Schmera, Heino, Podani, Erős, \& Dolédec, 2017).

Son múltiples los factores que inciden sobre los macroinvertebrados en un río o arroyo de montaña. El ambiente físico es un factor fundamental, donde el sustrato y la dinámica hidráulica modifican la disponibilidad de recursos y la estructura de las poblaciones (Death \& Winterbourn, 1995; Rempel, Richardson \& Healey, 2000; Brooks et al., 2005; Mesa, 2010; Vimos-Lojano et al., 2019). La materia orgánica según la fuente, su forma y calidad son el otro factor de gran relevancia en la estructura de estos ensamblajes, especialmente en sistemas lóticos andinos (Lujan, Roach, Jacobsen, Winemiller, Vargas, Ching \& Maestre, 2013).

Los macroinvertebrados poseen mecanismos de respuesta, conocidos como rasgos funcionales. Estos se definen como las características morfológicas, fisiológicas o ecológicas de los organismos que repercuten en su crecimiento, reproducción y supervivencia (Violle, Navas, Vile, Kazakou, Fortune, Hummel, \&
Garnier, 2007; Reynaga \& Dos Santos, 2012). El estudio de los rasgos representa una gran aplicabilidad en biomonitoreo, conservación y restauración de ecosistemas lóticos a nivel global, siendo un enfoque complementario que permite aproximarse objetivamente a la comprensión de los roles ecológicos y por ende al funcionamiento y estado del ecosistema (Bonada et al., 2006; Schmera et al., 2017). El estudio de los rasgos biológicos y su relación con la expresión y variabilidad del entorno es la base de la diversidad funcional, entendida como el valor y el rango de aquellos componentes de la biodiversidad que determinan como opera o funciona un ecosistema (Tilman, 2001). Si bien su medición es compleja y se conocen múltiples formas de aproximarse a ella (Petchey, Hector \& Gaston 2004; Ricotta, 2005), es importante considerar el efecto diferencial que puede tener cada población según su abundancia (Villéger, Mason \& Mouillot, 2008).

Los estudios de macroinvertebrados en Colombia han adicionado en los últimos años un enfoque funcional, sin embargo, estos son escasos en comparación con lo reportado en Norteamérica y Europa (Richards, Haro, Johnson, \& Host, 1997; Lamouroux, Dolédec, \& Gayraud, 2004; Heino, 2005; Bonada, Doledec, \& Statzner, 2007; Schmera et al., 2017). La mayoría de estos estudios se enfocan en las estrategias alimentarias de la comunidad y se concentran en la región Pacífica, Caribe y en menor proporción en la región Andina (e.g., Chará-Serna, Chará, Zúñiga, \& Pearson, 2012; Guzmán-Soto \& Tamarís-Turizo, 2014; Longo \& Blanco, 2014; Motta, Longo, \& ArangurenRiaño, 2017; Villada, Triana, \& Dias, 2017). El objetivo de este estudio fue determinar la estructura, diversidad taxonómica y diversidad funcional del ensamblaje de macroinvertebrados bajo diferentes expresiones físicas en el río Hato Laguna, tributario del Lago de Tota. Se planteó como hipótesis de investigación: el ensamblaje presentará una mayor diversidad taxonómica en los sitios donde existan mayor heterogeneidad espacial; por otra parte, se espera que la diversidad funcional esté relacionada con la diversidad taxonómica, sin 
embargo, se harán evidentes los momentos y condiciones que promueven la diversificación de rasgos morfo-fisiológicos como respuesta a las diferentes expresiones del hábitat. Nuestra predicción es que, en el tramo donde la velocidad, profundidad y rugosidad sean altas, predominarían taxones de tamaño pequeño, formas de cuerpo flexible, adaptaciones de sujeción al sustrato, grupo funcional alimenticio filtrador. Por otro lado, el tamaño máximo, cuerpo cilíndrico, tolerancia moderada al déficit de oxígeno, endobentónicos y grupo funcional alimenticio colectores-recolectores se expresarían más en el tramo con bajos valores de velocidad y rugosidad.

\section{MATERIALES Y MÉTODOS}

Área de Estudio: El estudio fue realizado en el río Hato Laguna (longitud $13 \mathrm{Km}$ y área de drenaje de $34 \mathrm{Km}^{2}$ ), tributario de la cuenca del Lago de Tota, en la Cordillera Oriental de los andes colombianos (CONPES 3801, 2014;
Plazas, 2016). El patrón de lluvias es de tipo bimodal con una precipitación media anual entre 625 y $825 \mathrm{~mm}$; con periodos máximos en los meses abril-mayo y octubre-noviembre, humedad relativa anual del $83 \%$ y temperatura media multianual de $11{ }^{\circ} \mathrm{C}$ (González \& Cañón, 2016; Wanumen, 2019). La vegetación está constituida por relictos de bosque altoandino, y plantaciones de especies de aliso (Alnus sp.), pino (Pinus patula), eucalipto (Eucaliptus globulus) entre otras especies exóticas debido a los diferentes procesos de transformación antrópica (Moreno, 2015; Plazas, 2016).

Diseño de muestreo: Se realizaron cuatro muestreos en época de bajas precipitaciones entre noviembre del 2017 y febrero del 2018 (Fig. 1), en dos tramos: tramo alto (TA) ( $\left.5^{\circ} 37^{\prime} 2^{\prime \prime} \mathrm{N} \& 72^{\circ} 52^{\prime} 35.2^{\prime \prime}\right)$ (Altitud: 3332 msnm), con presencia de vegetación de ribera tipo arbórea con extensión de $30 \mathrm{~m}$ de ancho y tramo bajo (TB) $\left(5^{\circ} 35^{\prime} 18.6^{\prime \prime} \mathrm{N} \& 72^{\circ} 53^{\prime}\right.$ 34.5 " W) (Altitud: $3042 \mathrm{msnm}$ ) con presencia

300

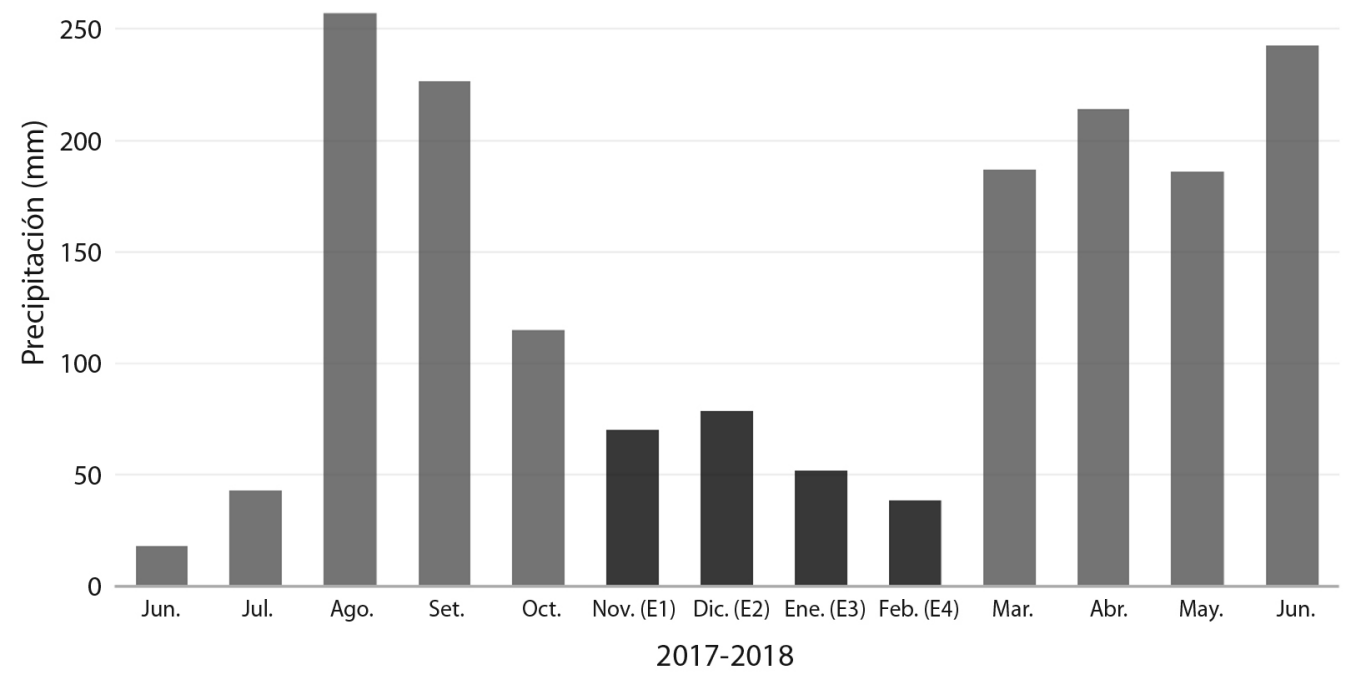

Fig. 1. Valores totales de precipitación mensuales (mm). En oscuro se muestran los meses de muestreo: noviembre (E1)diciembre (E2) 2017 y enero (E3) -febrero (E4) 2018. Tomado de: Instituto de Hidrología, Meteorología y Estudios Ambientales (IDEAM).

Fig. 1. Total monthly precipitation (mm). In darker color, sampling months are shown: November (E1) -December (E2) 2017 and January (E3) -February (E4) 2018. Taken from: Institute of Hydrology, Meteorology and Environmental Studies (IDEAM). 
de vegetación de ribera tipo herbácea, arbustiva y cultivos aledaños de cebolla larga (Allium fistulosum), papa (Solanum tuberosum), arveja (Pisum sativum).

En cada tramo (300 metros) se seleccionaron 15 puntos de muestreo de forma aleatoria estratificada: se estimó visualmente la proporción de cada tipo de mesohábitats presente para el cálculo del número de muestras biológicas a recolectarse de forma aleatoria en los mismos. Se realizó este tipo de muestreo ya que según Roleček, Chytrý, Hájek, Lvončík \& Tichý, (2007), en medios ambientes heterogéneos se obtiene una alta representación de la variabilidad espacial.

Variables físicas: En cada tramo se determinó la proporción de los siguientes mesohábitats: rápido (alta-velocidad y flujo-turbulento), corriente (alta-velocidad y flujo laminar), poza (nula o baja velocidad de flujo) (Parasiewicz, 2007). Se midió el ancho húmedo del canal en tres puntos dentro del tramo, y por cada muestra biológica se estimaron: el tipo de sustrato como bloque (diámetro $>1 \mathrm{~m}$ ), canto (diámetro 1-0.5 m), guijarro (diámetro $0.5-0.2 \mathrm{~m}$ ) y grava (diámetro 0.2-0.05 m) arenas (diámetro 0.005$0.002 \mathrm{~m}$ ) y limo (diámetro $<0.002 \mathrm{~m}$ ) (Apéndice 1) (Wadeson, 1994). La velocidad media de flujo $(\mathrm{m} / \mathrm{s})$ y la profundidad $(\mathrm{m})$ se midieron con un Sensor Hidrometric OTT. La rugosidad se calculó mediante la proporción entre la longitud lineal/ longitud contorno del sustrato. A partir de estos valores se estableció el índice de rugosidad, en el que un mayor valor indica una menor rugosidad y un menor valor una mayor rugosidad (Motta, Obregón \& Vimos, 2018).

Variables biológicas: Se recolectaron 15 muestras por tramo $(n=2)$ y por muestreo $(n=4)$ con un $\mathrm{N}$ total de 120 , utilizando una red Surber (área de $0.09 \mathrm{~m}^{2}$, abertura de malla de 300 $\mu \mathrm{m})$, por un tiempo de esfuerzo de 1 minuto cada muestra. El material recolectado fue preservado con etanol al $70 \%$ y fue transportado al laboratorio donde los macroinvertebrados fueron separados del resto del material, realizando la determinación al mayor nivel de resolución taxonómica posible, mediante claves especializadas como las de: Pennak (1989), Merrit et al. (2008), Domínguez \& Fernández (2009). Los especímenes fueron depositados en la colección limnológica de la Universidad Pedagógica y Tecnológica de Colombia (UPTC) con permiso de colecta dado en la resolución No. 01744 Autoridad Nacional De Licencias Ambientales (ANLA).

Rasgos funcionales: Los rasgos definidos abarcan seis categorías con sus respectivas modalidades; incluyen características morfológicas, fisiológicas y tróficas (Tabla 1). Se seleccionaron estas categorías de acuerdo con la posible relación con las variables físicas (Bonada, Rieradevall \& Prat, 2007; Tomanova \& Usseglio-Polatera, 2007). La asignación de los rasgos de: gremio trófico y largo del cuerpo se realizaron mediante mediciones directas de los taxones más abundantes $(>50$ individuos en total), el resto de taxones, al igual que los rasgos forma del cuerpo, tipo de respiración, movilidad y grupos funcionales alimenticios se obtuvieron de información disponible de Tachet, Richoux, Bournaud, \& Usseglio-Polatera, (2002); Tomanova \& Usseglio-Polatera (2007); Chará-Serna, Chará \& Zuñiga, (2012); Longo \& Blanco, (2014).

Para las categorías de gremio trófico, se examinó el tracto digestivo de 1038 organismos según lo propuesto por Muñoz, Romaní, Rodrigues, González, \& García, (2009). Así, por cada taxón se realizó la disección de mínimo diez individuos y se procedió bajo un estereoscopio a extraer el tubo digestivo; el contenido se colocó en una placa de forma homogénea para su posterior observación en el microscopio. Por último, se seleccionaron 15 campos al azar, teniendo como área definida el cubreobjetos y se identificó de forma visual por medio de una reglilla ocular micrométrica las siguientes categorías: material inorgánico, materia orgánica particulada fina (MOPF) (tamaño $<50 \mu \mathrm{m}$ ), materia orgánica particulada gruesa (MOPG) $(50 \mu \mathrm{m}-1 \mathrm{~mm})$, micrófitas, macrófitas y restos de animales. Finalmente, se estableció la proporción de cada categoría 
TABLA 1

Rasgos funcionales aplicados en el estudio con sus categorías y códigos

TABLE 1

Functional features applied in the study with its category and codes

\begin{tabular}{|c|c|c|}
\hline Rasgo & Categoría & Código \\
\hline \multirow{7}{*}{$\begin{array}{l}\text { Largo del cuerpo } \\
(\mathrm{mm})\end{array}$} & $<2.5$ & LA \\
\hline & $2.5-5$ & LB \\
\hline & $5-10$ & $\mathrm{LC}$ \\
\hline & $10-20$ & LD \\
\hline & $20-40$ & LE \\
\hline & $40-80$ & $\mathrm{LF}$ \\
\hline & $>80$ & LG \\
\hline \multirow[t]{4}{*}{ Forma del cuerpo } & Cilíndrico & Cil \\
\hline & Esférico & Esf \\
\hline & Aplanado & Apla \\
\hline & Optimizado & Opt \\
\hline \multirow[t]{4}{*}{ Respiración } & Branquias & Bran \\
\hline & Plastrón & Plas \\
\hline & Tegumento & Teg \\
\hline & Estigma & Est \\
\hline \multirow[t]{7}{*}{ Movilidad } & Volador & Vol \\
\hline & Nadador Superficial & N Sup \\
\hline & Nadador Profundo & N Pro \\
\hline & Rastrero & Rast \\
\hline & Epibentónico & EpB \\
\hline & Endobentónico & EnB \\
\hline & Unido al Sustrato & U Sust \\
\hline \multirow[t]{6}{*}{ Gremio Trófico } & Material Inorgánico & M Ing \\
\hline & $\begin{array}{l}\text { Materia orgánica } \\
\text { particulada fina }\end{array}$ & MOPF \\
\hline & $\begin{array}{l}\text { Materia orgánica } \\
\text { particulada gruesa }\end{array}$ & MOPG \\
\hline & Micrófitas & Micro \\
\hline & Macrófitas & Macro \\
\hline & Animales & Ani \\
\hline \multirow{5}{*}{$\begin{array}{l}\text { Grupo Funcional } \\
\text { Alimenticio }\end{array}$} & Colector & CL \\
\hline & Triturador & TR \\
\hline & Raspador & $\mathrm{RS}$ \\
\hline & Filtrador & FL \\
\hline & Depredador & DP \\
\hline
\end{tabular}

Basado en Cummins, 1973, Tachet et al., 2002, Bonada et al., 2007 y Tomanova \& Usseglio-Polatera, 2007.

en los campos observados (Apéndice 3). Dado que las asignaciones de estas categorías en su mayoría están adaptadas a los ecosistemas norteamericanos y europeos; se evaluó el contenido estomacal para complementar o confirmar la información de la literatura de referencia.

Análisis estadístico: Las variables físicas se analizaron realizando una ANOVA no paramétrica de Kruskal-Wallis (Guisande, Barreiro, Maneiro, Riveiro, \& Vergara, 2006), para identificar posibles diferencias entre los tramos utilizando el programa PAST v.3.13; las variables fueron estandarizadas según lo propuesto por Guisande et al. (2006).

Se evaluó la variación en la composición y estructura de la comunidad de macroinvertebrados entre los tramos y muestreos mediante el análisis de similitud (ANOSIM) basado en Bray-Curtis, con 9999 permutaciones en el programa PAST versión 3.13 (Clarke, 1993). Para aquellos casos en que se encontraron diferencias significativas mediante el análisis de similitud, se realizó un análisis de porcentaje de similitud (SIMPER) con el propósito de identificar las especies responsables de la diferenciación.

La representatividad de las muestras para hacer estimaciones de diversidad se evaluó mediante análisis de cobertura (Apéndice 4) (Chao \& Jost, 2012):

$$
\hat{\mathrm{C}}_{\mathbf{n}}=1-\frac{f 1}{n}\left[\frac{(n-1) f 1}{(n-1) f 1+2 f 2}\right]
$$

donde:

$\mathrm{n}=$ Abundancia Total

$f 1=$ Número de singletons (especies que tienen solo un individuo en la muestra de referencia)

Para estimar la diversidad taxonómica de los macroinvertebrados por tramos y muestreos, se calcularon los índices de diversidad de Simpson (1-D), índice de Shannon Wiener $\left(\mathrm{H}^{\prime}\right)$ y el índice de equidad de Pielou $\left(\mathrm{J}^{\prime}\right)$ (Moreno, 2001).

En cuanto a los rasgos funcionales, se construyó una matriz de los taxones con cada una de las categorías de los rasgos, estableciendo una codificación difusa: 0 (no tiene ningún vínculo), 1 (vínculo débil), 2 (vínculo moderado), 3 (vínculo fuerte); este enfoque 
integra la plasticidad de cada taxón teniendo en cuenta que este puede vincularse a más de una categoría por rasgo (Chevenet, Doléadec, \& Chessel, 1994). Luego, esta matriz fue multiplicada por la matriz de las abundancias de los taxones con el fin de obtener una matriz final de los rasgos por tramo y muestreo. Con esta información se realizó un Análisis de Correspondencia Difusa (ACD) y un Análisis de Componentes Principales Difuso (ACPD) en el programa Rstudio versión 1.2.1335, con los cuales se determinó la relación de dependencia entre los rasgos funcionales y la variación temporal. Posteriormente, se identificaron los rasgos funcionales que presentaron diferencias significativas en el espacio y el tiempo, por medio de la prueba de Fisher.

La diversidad funcional se estimó como medidas del espacio funcional ocupado por las especies según la expresión de los rasgos, se consideró la riqueza funcional (RF), la divergencia funcional (DF) y la equidad funcional (EF) como descriptores (Villéger, et al., 2008). Los rasgos seleccionados según las categorías y codificación presentados en la tabla $1 \mathrm{y}$ las abundancias relativas obtenidas a partir de los valores absolutos presentados en la tabla 2 . Los descriptores de diversidad funcional fueron estimados mediante el paquete FD (Laliberté, Legendre, Shipley, Laliberté, 2014) en el software Rstudio versión 1.2.1335 (R Development Core Team, 2019)

Para establecer las relaciones entre las variables físicas y los rasgos funcionales se realizó un Análisis de Correspondencia Canónica (ACC) empleando la matriz de rasgo por tramo y muestras y los datos físicos estandarizados. Previo al uso del análisis, se exploraron los datos mediante DCA (Análisis de correspondencias sin tendencias) para estimar la longitud de la gradiente, la cual fue de un gradiente 1.9 unidades de desviación estándar, correspondiente a un modelo unimodal seleccionado para el análisis (Lepŝ \& Ŝmilauer, 2003). Por otro lado, las abundancias de individuos por cada taxón fueron transformados con $\log 10(\mathrm{x}+1)$ y se eliminaron los taxones raros (abundancias $<10$ individuos del total) para el análisis. Para la validación del mismo se utilizó la prueba de permutación de Monte Carlo con 499 permutaciones. Además, se verificó que las variables físicas no estuvieran correlacionadas (Spearman: $\rho>|0.8|)$. Por último, se aplicó la corrección de Bonferroni con el fin de evitar el error de tipo I en los datos. Este análisis se realizó con el programa CANOCO 5.

\section{RESULTADOS}

Las variables físicas mostraron una variación entre tramos y muestreos. La velocidad del flujo fue mayor en el tramo alto (Kruskal Wallis test: $\mathrm{H}=0.162$, gl1=7, $p<0.05$ ), mientras que el ancho del canal fue mayor en el tramo bajo (Kruskal Wallis test: $\mathrm{H}=0.001$, $\mathrm{gll}=7, p<0.05)$. Por otro lado, el ancho del canal (Kruskal Wallis test: $\mathrm{H}=17.7, \mathrm{gll}=3, p$ $<0.001$ ) y la profundidad (Kruskal Wallis test: $\mathrm{H}=24.530$, gl1 $=3, p<0.001$ ) tuvieron valores mayores en el muestro E1. Además, se observó que los valores de velocidad y la profundidad tuvieron un alto coeficiente de variación dentro de los tramos $(>50 \%)$, respecto a los valores del índice de rugosidad y el ancho del canal $(<20 \%)$ (Tabla 2).

Respecto de los macroinvertebrados, se recolectaron 4531 individuos distribuidos en 38 taxones. El orden más diverso fue Diptera, distribuido en nueve familias, donde Chironomidae fue la más diversa, con el $86 \%$ de representación dentro del orden. Asimismo, la subfamilia de quironómidos Orthocladiinae presentó la mayor abundancia. Seguidamente, el orden Coleoptera estuvo representado por cuatro familias de las cuales la más diversa fue Elmidae con cuatro géneros. Por otro lado, el orden Trichoptera se registró en todos los tramos y muestreos con dos familias y dos géneros (Tabla 3). La composición y abundancia no mostraron diferencias significativas entre los tramos (ANOSIM, $\mathrm{R}=0.037, p=$ 0.002) y muestreos $(\mathrm{R}=0.167, \mathrm{p}=0.0001)$. Por otra parte, se resaltó la variación en la abundancia entre tramos de algunos taxones como Orthocladiinae sp7 $(\mathrm{TA}=731, \mathrm{~TB}=54$ Individuos $\left./ \mathrm{m}^{2}\right)$, Nectopsyche $\mathrm{sp} .(\mathrm{TA}=48, \mathrm{~TB}$ 
TABLA 2

Variables físicas registradas en el río Hato Laguna, tributario del Lago de Tota. Donde X, $\alpha$ y CV representan el promedio, desviación estándar y el coeficiente de variación, respectivamente. Las siglas en la columna izquierda indican la combinación del tramo $(\mathrm{TA}=$ tramo alto; $\mathrm{TB}=$ tramo bajo $)$ y el momento de muestreo $(\mathrm{E} 1=$ noviembre; $\mathrm{E} 2=$ diciembre; $\mathrm{E} 3=$ enero; $\mathrm{E} 4=$ febrero $)$

TABLE 2

Physical variables registered in the Hato Laguna river, tributary of Lake Tota. Where X, $\alpha$ and CV represent the average, standard deviation and the coefficient of variation, respectively. The acronyms in the left column indicate the combination of the section $(\mathrm{TA}=$ high section; $\mathrm{TB}=$ low section) and the sampling events $(\mathrm{E} 1$ = November; E2 = December; E3 = January; E4 = February $)$

\begin{tabular}{lccccccccccccc} 
& \multicolumn{3}{c}{ Velocidad $(\mathrm{m} / \mathrm{s})$} & \multicolumn{4}{c}{ Profundidad $(\mathrm{m})$} & \multicolumn{3}{c}{ Índice de rugosidad } & \multicolumn{3}{c}{ Ancho (m) } \\
& $\mathrm{X}$ & $\alpha$ & $\mathrm{CV}$ & $\mathrm{X}$ & $\alpha$ & $\mathrm{CV}$ & $\mathrm{X}$ & $\alpha$ & $\mathrm{CV}$ & $\mathrm{X}$ & $\alpha$ & $\mathrm{CV}$ \\
TAE1 & 0.532 & 0.377 & 70.917 & 0.214 & 0.192 & 89.512 & 0.781 & 0.105 & 13.471 & 5.076 & 0.461 & 9.089 \\
TBE1 & 0.382 & 0.225 & 58.866 & 0.183 & 0.106 & 57.833 & 0.793 & 0.080 & 10.134 & 4.490 & 0.442 & 9.852 \\
TAE2 & 0.372 & 0.357 & 95.942 & 0.167 & 0.129 & 77.101 & 0.844 & 0.111 & 13.179 & 3.387 & 0.534 & 15.778 \\
TBE2 & 0.264 & 0.213 & 80.670 & 0.214 & 0.075 & 35.166 & 0.855 & 0.098 & 11.445 & 3.245 & 0.667 & 20.549 \\
TAE3 & 0.304 & 0.307 & 101.121 & 0.096 & 0.087 & 91.444 & 0.904 & 0.098 & 10.796 & 3.307 & 0.363 & 10.991 \\
TBE3 & 0.188 & 0.275 & 145.932 & 0.149 & 0.172 & 115.44 & 0.853 & 0.114 & 13.320 & 3.768 & 0.398 & 10.569 \\
TAE4 & 0.544 & 0.421 & 77.332 & 0.072 & 0.045 & 62.234 & 0.809 & 0.136 & 16.747 & 2.490 & 0.208 & 8.335 \\
TBE4 & 0.287 & 0.295 & 103.067 & 0.082 & 0.071 & 86.391 & 0.832 & 0.140 & 16.862 & 3.590 & 0.562 & 15.650 \\
Kruskal-Wallis & $\mathrm{H}$ & $\mathrm{gl}$ & $p$ & $\mathrm{H}$ & $\mathrm{gl}$ & $p$ & $\mathrm{H}$ & $\mathrm{Gl}$ & $p$ & $\mathrm{H}$ & $\mathrm{gl}$ & $p$ \\
Tramos & 0.162 & 7 & $<0.05$ & 0.232 & 7 & 0.450 & 0.137 & 7 & 0.210 & 0.001 & 7 & $<0.05$ \\
Muestreo & 3.560 & 3 & 0.312 & 24.530 & 3 & $<0.001$ & 1.520 & 3 & 0.676 & 17.700 & 3 & $<0.001$ \\
\hline
\end{tabular}

Promedio (X), Desviación Estándar ( $\alpha$ ), Coeficiente de Variación (CV).

$=232$ Individuos $\left./ \mathrm{m}^{2}\right)$, Andesiops sp. $(\mathrm{TA}=87$, $\mathrm{TB}=22$ Individuos $\left./ \mathrm{m}^{2}\right)$ y Simulium $\mathrm{sp}$. $(\mathrm{TA}=$ $167, \mathrm{~TB}=76$ Individuos $/ \mathrm{m}^{2}$ ) entre muestreos los taxones Orthocladiinae sp2 $(\mathrm{E} 1=418, \mathrm{E} 2=$ 95, E3= 70 y $\mathrm{E} 4=322$ Individuos $/ \mathrm{m}^{2}$ ), Orthocladiinae sp4 $(\mathrm{E} 1=301, \mathrm{E} 2=305, \mathrm{E} 3=153 \mathrm{y}$ $\mathrm{E} 4=419$ Individuos $/ \mathrm{m}^{2}$ ) (Tabla 3).

Respecto a la diversidad, la cobertura de la muestra estimada fue superior al $97 \%$ en todos los casos, lo que permite mayor confiabilidad en los estimadores de diversidad (Apéndice 4). Así, los resultados de los índices de Simpson, Shannon y Pielou, muestran variación en la estructura taxonómica de los macroinvertebrados entre tramos y entre muestreos, sin que se logre identificar un patrón o tendencia en su expresión (Tabla 4).

En cuanto a los rasgos funcionales, en general se resalta la dominancia de ciertas categorías tales como: longitud del cuerpo entre 20-40 mm, forma del cuerpo cilíndrico, respiración por branquias, la categoría de movilidad rastrero y epibentónico, tipo de alimentación de MOPF y MOPG, y el grupo funcional alimenticio colector y filtrador.

Según la prueba de Fisher las categorías que presentaron diferencias significativas $(p<$ 0.05 ) entre tramos y muestreos fueron; largo del cuerpo de 20 a $40 \mathrm{~mm}$ (tramos), forma del cuerpo cilíndrico (muestreos), respiración por branquias (tramos), movilidad de tipo rastrero, gremio trófico MOPF y grupo funcional alimenticio colector

En el ACPD el porcentaje de inercia proyectado en el eje uno fue de $60.64 \%$ y en el eje dos de $18.24 \%$. Según este análisis, el tramo alto en los muestreos (E2 y E4) se asoció con los rasgos; largo del cuerpo 5 a $10 \mathrm{~mm}$, respiración por tegumento, gremio trófico MOPF y grupo funcional alimenticio colector (Fig. 2, Lado derecho). El tramo bajo en el muestreo (E2) y tramo alto (E3) se asociaron con tamaño del cuerpo de 10-20 mm, gremio trófico MOPG, grupo funcional alimenticio triturador 
TABLA 3

Lista de taxones y abundancia absoluta (Individuos $/ \mathrm{m}^{2}$ ) de los macroinvertebrados en el río Hato Laguna en el Tramo Alto (TA) y Tramo Bajo (TB) y momentos de muestreo (E1, E2, E3 y E4)

TABLE 3

List of taxa and absolute abundance (Individuals $/ \mathrm{m}^{2}$ ) of the macroinvertebrates in the Hato Laguna river in the High Section (TA) and Low Section (TB) and sampling events (E1, E2, E3 and E4)

\begin{tabular}{|c|c|c|c|c|c|c|c|c|c|c|}
\hline Orden & Familia & Taxón & TAE1 & TBE1 & TAE2 & TBE2 & TAE3 & TBE3 & TAE4 & TBE4 \\
\hline \multirow[t]{3}{*}{ Haplotaxida } & Lumbriculidae & Lumbriculus sp. & 1 & 16 & 0 & 0 & 0 & 0 & 0 & 3 \\
\hline & Tubificidae & Tubificidae & 0 & 0 & 1 & 3 & 0 & 7 & 5 & 16 \\
\hline & Naididae & Sthephensoniana sp. & 14 & 9 & 0 & 1 & 0 & 0 & 0 & 19 \\
\hline \multirow[t]{2}{*}{ Ephemeroptera } & Baetidae & Andesiops sp. & 75 & 12 & 10 & 8 & 2 & 1 & 0 & 1 \\
\hline & & Baetodes sp. & 3 & 36 & 34 & 9 & 0 & 1 & 5 & 4 \\
\hline Odonata & Aeshnidae & Aeshnidae & 1 & 0 & 0 & 0 & 0 & 0 & 0 & 0 \\
\hline \multirow[t]{2}{*}{ Trichoptera } & Hydrobiosidae & Atopsyche sp. & 6 & 17 & 4 & 2 & 5 & 0 & 2 & 2 \\
\hline & Leptoceridae & Nectopsyche sp. & 8 & 39 & 9 & 87 & 27 & 12 & 4 & 94 \\
\hline \multirow[t]{17}{*}{ Diptera } & Blephariceridae & Blepharicera sp. & 0 & 0 & 1 & 0 & 0 & 0 & 0 & 1 \\
\hline & Ceratopogonidae & Ceratopogonidae & 0 & 0 & 1 & 1 & 0 & 17 & 11 & 91 \\
\hline & Chironomidae & Chironominae & 0 & 3 & 2 & 5 & 0 & 15 & 0 & 21 \\
\hline & & Orthocladiinae sp1 & 19 & 77 & 0 & 0 & 0 & 0 & 0 & 0 \\
\hline & & Orthocladiinae sp2 & 120 & 298 & 27 & 68 & 27 & 43 & 202 & 120 \\
\hline & & Orthocladiinae sp3 & 1 & 103 & 3 & 56 & 6 & 5 & 4 & 12 \\
\hline & & Orthocladiinae sp4 & 87 & 214 & 206 & 99 & 54 & 57 & 149 & 270 \\
\hline & & Orthocladiinae sp6 & 2 & 37 & 0 & 3 & 0 & 0 & 0 & 15 \\
\hline & & Orthocladiinae sp7 & 95 & 3 & 336 & 7 & 13 & 0 & 287 & 44 \\
\hline & Tanypodinae & Tanypodinae & 2 & 7 & 7 & 1 & 9 & 3 & 30 & 3 \\
\hline & Empididae & Empididae & 4 & 0 & 0 & 0 & 0 & 0 & 7 & 2 \\
\hline & Muscidae & Muscidae & 0 & 0 & 0 & 1 & 0 & 1 & 0 & 1 \\
\hline & Sciomyzidae & Sciomyzidae & 1 & 1 & 0 & 1 & 0 & 0 & 0 & 0 \\
\hline & Simuliidae & Simulium sp. & 61 & 23 & 68 & 30 & 5 & 3 & 36 & 10 \\
\hline & Tipulidae & Hexatoma sp. & 2 & 1 & 4 & 7 & 0 & 1 & 3 & 16 \\
\hline & & Limonia $\mathrm{sp}$. & 0 & 1 & 3 & 0 & 0 & 2 & 0 & 1 \\
\hline & & Tipula sp. & 3 & 7 & 1 & 0 & 2 & 0 & 3 & 0 \\
\hline \multirow[t]{8}{*}{ Coleoptera } & Elmidae & Heterelmis sp. (Adt) & 0 & 0 & 0 & 1 & 0 & 0 & 0 & 0 \\
\hline & & Neoelmis sp.(Adt) & 9 & 4 & 6 & 4 & 26 & 0 & 4 & 3 \\
\hline & & Neoelmis sp.(larv) & 10 & 1 & 1 & 1 & 3 & 1 & 2 & 20 \\
\hline & & Hexacylloepus sp. (Adt) & 0 & 0 & 2 & 0 & 2 & 0 & 0 & 0 \\
\hline & & Hexacylloepus sp.(Larv) & 8 & 0 & 4 & 14 & 7 & 4 & 5 & 8 \\
\hline & Ptilodactylidae & Ptilodactylidae & 1 & 0 & 0 & 0 & 0 & 0 & 0 & 0 \\
\hline & Scirtidae & Scirtes sp. & 0 & 1 & 2 & 0 & 3 & 0 & 2 & 0 \\
\hline & Staphylinidae & Staphylinidae & 0 & 0 & 0 & 8 & 1 & 0 & 5 & 0 \\
\hline Neoophora & Dugesiidae & Dugesia $\mathrm{sp}$. & 1 & 2 & 0 & 0 & 0 & 0 & 4 & 5 \\
\hline Amphipoda & Hyalellidae & Hyallela $\mathrm{sp}$. & 0 & 12 & 0 & 1 & 1 & 2 & 0 & 2 \\
\hline Isopoda & Sphaeromatidae & Sphaeroma sp. & 0 & 0 & 1 & 0 & 0 & 0 & 0 & 0 \\
\hline \multicolumn{3}{|c|}{ Abundancia Total } & 534 & 924 & 733 & 418 & 193 & 175 & 770 & 784 \\
\hline \multicolumn{3}{|l|}{ Riqueza Total } & 24 & 24 & 23 & 24 & 17 & 17 & 20 & 26 \\
\hline
\end{tabular}


TABLA 4

Índices de diversidad taxonómica aplicados al ensamblaje de macroinvertebrados en el río Hato Laguna. Índice de Simpson (1-D), Índice de Diversidad de Shannon-Wiener ( $\left.\mathrm{H}^{\prime}\right)$ e Índice de Equidad de Pielou ( $\left.\mathrm{J}^{\prime}\right)$. Las siglas en la columna izquierda indican la combinación del tramo $(\mathrm{TA}=$ tramo alto; $\mathrm{TB}=$ tramo bajo) y el momento de muestreo $(\mathrm{E} 1=$ noviembre; $\mathrm{E} 2=$ diciembre; $\mathrm{E} 3=$ enero; $\mathrm{E} 4=$ febrero $)$

TABLE 4

Taxonomic diversity indices applied to the macroinvertebrate assemblages in the Hato Laguna river. Simpson Index (1-D), Shannon-Wiener Diversity Index $\left(\mathrm{H}^{\prime}\right)$ and Pielou Equity Index $\left(\mathrm{J}^{\prime}\right)$. The acronyms in the left column indicate the combination of the section $(\mathrm{TA}=$ high section; $\mathrm{TB}=$ low section $)$ and the sampling events $(\mathrm{E} 1$ = November; E2 = December; E3 = January; E4 = February $)$

\begin{tabular}{cccccccc} 
Tramo Alto & Simpson $(1-\mathrm{D})$ & Shannon $\left(\mathrm{H}^{\prime}\right)$ & Pielou $\left(\mathrm{J}^{\prime}\right)$ & Tramo Bajo & Simpson $(1-\mathrm{D})$ & Shannon $\left(\mathrm{H}^{\prime}\right)$ & Pielou $\left(\mathrm{J}^{\prime}\right)$ \\
TAE1 & 0.856 & 2.231 & 0.702 & TBE1 & 0.816 & 2.118 & 0.667 \\
TAE2 & 0.696 & 1.615 & 0.515 & TBE2 & 0.847 & 2.206 & 0.694 \\
TAE3 & 0.853 & 2.243 & 0.792 & TBE3 & 0.808 & 2.04 & 0.72 \\
TAE4 & 0.752 & 1.748 & 0.584 & TBE4 & 0.823 & 2.203 & 0.676 \\
\hline
\end{tabular}

(Fig. 2, Lado izquierdo). Por otra parte, los tramos bajos en los muestreos (E3 y E4) presentaron mayor proporción de los rasgos: forma del cuerpo aplanado, respiración por branquias, gremio trófico micrófitas y grupo funcional alimenticio raspador (Fig. 2, Parte inferior central).

Los descriptores de diversidad funcional muestran diferencias entre tramos y muestreos (Fig. 3). La riqueza funcional es mayor en el tramo alto, aunque en E4, se expresan magnitudes similares entre los tramos, se destaca una reducción de la RF en el E3 para ambos tramos. La equidad funcional muestra una tendencia similar entre tramos, sin embargo, en el E4 este descriptor se incrementa en el tramo alto. Respecto a la divergencia funcional, el tramo alto presenta mayor expresión de DF consistente durante los cuatro muestreos.

Respecto al ACC de los rasgos funcionales y las variables físicas, se presentó una inercia total de $81 \%$, con un valor de significancia de ( $\mathrm{p}<0.05$ ). El primer eje explicó el $46 \%$ de la varianza y en el segundo el $35 \%$ (Fig. 3). El primer eje se relacionó con valores altos de ancho del canal ( $p<0.01,52.4 \%$ contribución) y velocidad ( $p<0.01,33.4 \%$ contribución), que a su vez se relacionaron con las categorías unido al sustrato, endobentónico y volador. Por otro lado, el segundo eje se relacionó con valores altos velocidad $(p<0.01,47.2 \%$ contribución), valores bajos de ancho del canal $(p<0.01,30.1 \%$ contribución), rugosidad ( $p<0.05,22.54 \%$ contribución) y con las categorías forma del cuerpo aplanado, tipo de alimentación micrófitas y tamaño del cuerpo mayor a $80 \mathrm{~mm}$ (Fig. 4).

\section{DISCUSIÓN}

Este estudio demostró que las variables físicas mostraron fluctuaciones entre tramos y muestreos. De esta forma, la variación en la velocidad y ancho del canal entre los tramos se puede explicar por los cambios a lo largo del río en la pendiente, geomorfología del canal y cantidad de flujo transportado (Vannote, Minshall, Cummins, Sedell, \& Cushing, 1980; Montgomery \& Buffington, 1998). Estas variaciones longitudinales generan, en los tramos altos de los ríos, condiciones hidráulicas heterogéneas en la profundidad y altas velocidades del flujo, mientras que en los tramos bajos disminuye la velocidad debido a una menor pendiente y aumento del ancho del canal por el incremento en la cantidad de flujo transportado (Allan \& Castillo, 2007; Elosegi \& Sabater, 2009). Por otro lado, se observó que a pesar de que todos los muestreos corresponden a épocas de bajas precipitaciones, algunas variables como ancho y profundidad cambiaron entre muestreos. Esto se puede explicar por el tamaño 


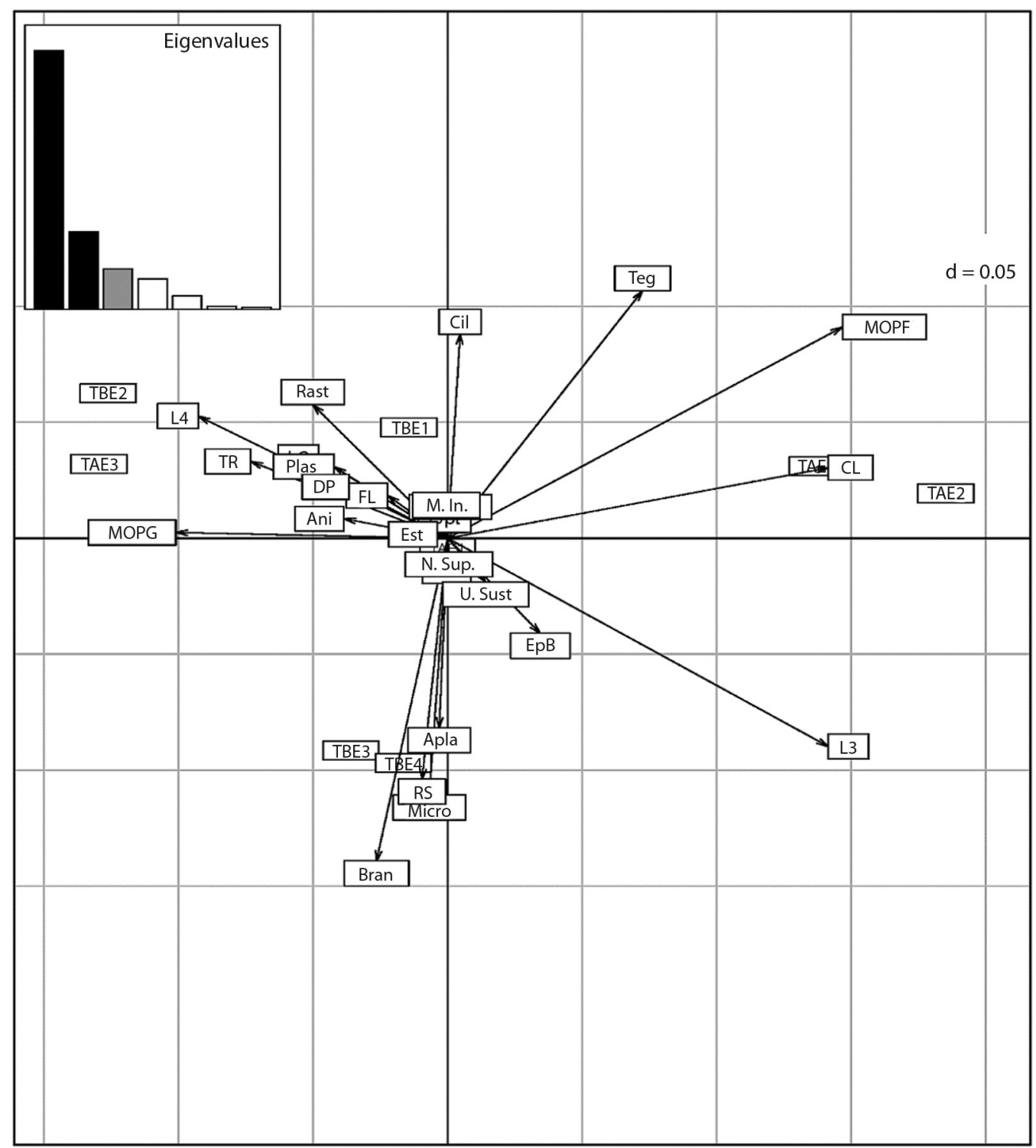

Fig. 2. Análisis de componentes principales difuso (ACPD) de las categorías de los rasgos en relación con la variación temporal. E1 (noviembre), E2 (diciembre), E3 (enero), E4 (febrero). Tramo Alto (TA), Tramo Bajo (TB). Ver códigos de los rasgos funcionales en la Tabla 1.

Fig. 2. Fuzzy Principal Component Analysis (ACPD) of the trait categories in relation to temporal variation. E1 (November), E2 (December), E3 (January), E4 (February). High Section (TA), Low Section (TB). See codes of functional trait in Table 1.

pequeño de la cuenca, que genera una respuesta rápida de aumento de flujo incluso con precipitaciones esporádicas, que se ve reflejado en el aumento del ancho y la profundidad en el muestreo E1 (noviembre) (IDEAM, 2018; Lewis, 2008).

En general, los órdenes Diptera, Coleoptera y Trichoptera mostraron la mayor riqueza taxonómica. Estos grupos se consideran 

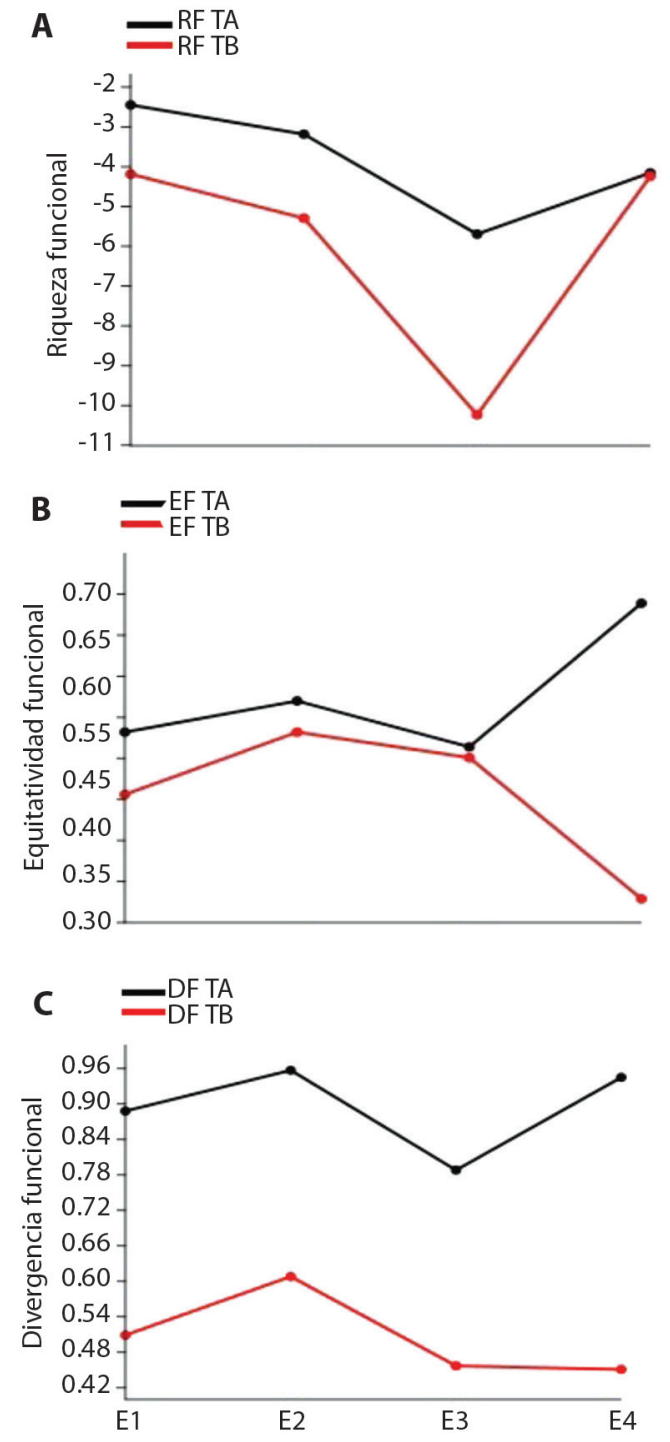

Fig. 3. Índices de diversidad funcional aplicados al ensamblaje de macroinvertebrados por tramos y muestreos. (A). Riqueza Funcional (RF), (B) Equidad Funcional (EF), (C) Divergencia Funcional (DF).

Fig. 3. Functional diversity indices applied to the macroinvertebrate assemblages by sections and samples. (A). Functional Richness (FR), (B) Functional Evenness (FE), (C) Functional Divergence (FD).

habituales en los sistemas de montaña andinos (Domínguez \& Fernández, 2009; Longo, Zamora, Guisande, \& Ramírez, 2010; Vásquez-Ramos \& Reinoso, 2012; Roldán, Zúñiga,

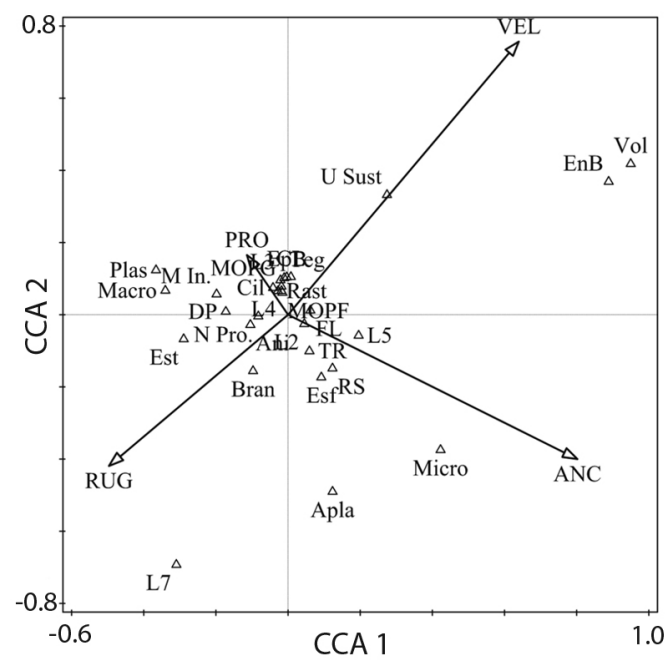

Fig. 4. Análisis de Correspondencia Canónica (ACC) entre los rasgos funcionales y las variables físicas. Velocidad (VEL), Rugosidad (RUG), Profundidad (PRO), Ancho (ANC). Varianza explicada, Componente 1 (horizontal): $46 \%$, Componente 2 (vertical): $35 \%$. Test de permutación Monte Carlo $(p=0.034)$. Ver códigos de los rasgos funcionales en la Tabla 1.

Fig. 4. Canonical Correspondence Analysis (CCA) between functional traits and physical variables. Speed (VEL), Roughness (RUG), Depth (PRO), Width (ANC). Variance explained, Component 1: 46\%, Component 2: 35\%. Monte Carlo permutation test $(\mathrm{p}=0.034)$. See codes of functional trait in Table 1 .

Zamora, Reinoso \& Longo, 2014). En cuanto a la estructura del ensamblaje, los taxones dominantes encontrados en el estudio pertenecen a la subfamilia Orthocladiinae (Chironomidae), reportados para ríos de alta montaña de Colombia, Ecuador y Perú (Prat, Ribera, Rieradevall, Villamarin, \& Acosta, 2013). Estos taxones son considerados abundantes en estos sistemas acuáticos y pioneros en la recolonización (Acuña, Muñoz, Giorgi, Omella, Sabater, \& Sabater, 2005), debido a su capacidad de dispersión, a sus hábitos tróficos generalistas y a sus adaptaciones para evitar las condiciones hidráulicas extremas relacionadas con su ciclo de vida o el tamaño del cuerpo pequeño que los favorece en la búsqueda de refugios para evitar la fuerza de arrastre de la corriente en velocidades altas (Matthaei, Arbuckle, \& Townsend, 2000; Henriques-Oliveira, Nessimian, 
\& Dorvillé, 2003; Mesa, 2010; Vimos-Lojano, 2017). Lo anterior pudo explicar su dominancia en los tramos altos donde la velocidad fue mayor pero también en menor proporción en los tramos bajos donde la velocidad disminuyó y aumentó el ancho de canal y la profundidad.

Por otra parte, la composición y la abundancia de los organismos no presentaron diferencias contrastantes entre tramos y muestreos según los análisis realizados. Sin embargo, se resalta la variación en la abundancia de taxones como los dípteros Orthocladiinae y Simulium sp., el efemeróptero Andesiops sp. y el tricóptero Nectopsyche sp. Este último, tuvo mayor abundancia en los tramos bajos en donde las velocidades de flujo fueron menores. Este hallazgo concuerda con lo reportado por Ríos-Touma, Encalada \& Prat. (2011) y VimosLojano, Martínez-Capel \& Hampel (2017) para sistemas de montaña andinos de Ecuador, en donde se demuestra que Nectopsyche sp. tiene una preferencia marcada por hábitats con bajas velocidades donde se acumula material de piedras y hojas en el lecho del río que es utilizado para la construcción de estuches característicos de este género y esta materia orgánica gruesa también puede ser aprovechada como recurso alimenticio por estos organismos trituradores (Holzenthal \& Ríos-Touma, 2018).

Por otro lado, Orthocladiinae, Simulium sp. y Andesiops sp. fueron más abundantes en los tramos altos. Este último, presenta un tamaño pequeño y uñas tarsales con dentículos que le confieren adherencia al sustrato y le permiten contrarrestar la fuerza de arrastre en condiciones de altas velocidades. (Domínguez \& Fernández, 2009) Asimismo, Simulium sp. se caracteriza por ser una especie reofílica, destacándose por su capacidad de adherencia al sustrato por medio de un anillo de ganchos anales y la utilización de un hilo de seda, siendo considerada una especie con movilidad epibentónica. (Pavitra, Low, Tan, Lim \& Ya'cob, 2019)

La diversidad taxonómica no expresó tendencias marcadas por tramos o muestreos, según Poff (1997) y Jacobsen (2004) esto puede explicarse porque estos estimadores están relacionados con patrones que se presentan a escalas espaciales mayores (segmento, río) como variaciones geomorfológicas, cambios en el tipo de vegetación y usos del suelo; así mismo, el estudio abarco cuatro meses, lo que limitaría ver cambios estacionales. (Villamarín, 2008). Sin embargo, Carrera \& Gunkel (2003); Rodríguez-Barrios, Ospina-Torres \& TurizoCorrea (2011), han encontrado que variaciones ambientales a escalas pequeñas en las condiciones físicas de velocidad, profundidad y ancho de canal como las halladas en el sistema de estudio, pueden incidir en la diversidad taxonómica. Por ejemplo, se ha reportado que un aumento en la velocidad y profundidad del flujo están relacionados con una menor diversidad de macroinvertebrados acuáticos en ríos de Chile y Argentina (Oyanedel, Valdovinos, Azócar, Moya, Mancilla, Pedreros \& Figueroa, 2008; Moya, Valdovinos, Moraga, Romero, Debels \& Oyanedel, 2009). Para el caso de estudio, en el tramo bajo con menor velocidad y mayor ancho del cauce, la tendencia a una mayor diversidad en los estimadores $\mathrm{H}^{\prime}$ y 1-D, está relacionada con mayor grado de equidad.

En cuanto a los rasgos funcionales, se destaca la dominancia generalizada de organismos colectores que se alimentan de MOPF. Estos hallazgos coinciden con lo reportado por Moretti, (2005), Tomanova, Goitia, \& Helešic, (2006) y Rivera, Zapata, Pérez, Morales, Ovalle, \& Alvarez (2010). Estos autores sugieren que la dominancia de este grupo en los ríos Neotropicales, se debe al aporte de MOPF derivada de la cuenca y/o de la descomposición por microorganismos y/o la abrasión física de la MOPG que posteriormente es acumulada por procesos de la hidráulica del río (Príncipe, Gualdoni, Oberto, Raffaini, \& Corigliano, 2010; Meza-S, Rubio-M, Dias, \& Walteros, 2012).

Las evidencias de variación en algunos rasgos funcionales, se observaron en el tramo alto en los muestreos de diciembre (E2) y febrero (E4) con aumento de la dominancia de los organismos que se alimentan de MOPF (colectores) como los Orthocladiinae, lo que se puede explicar por la alta velocidad del flujo en 
este tramo, dado por la pendiente que genera mayor transporte y posterior acumulación de sedimentos y materia orgánica particulada fina, fuente de alimento para los organismos colectores (Montgomery \& Buffington, 1998; Chará-Serna, Chará, Zuñiga, Pedraza \& Giraldo, 2010; Rolls, Leigh, \& Sheldon, 2012). Estos resultados sugieren que procesos descritos a gran escala como los propuesto para regiones templadas por Vannote et al. (1980), en los que los colectores dominan en la parte baja de la cuenca debido a la relevancia de procesos de depósitos de sedimentos, podría expresarse en pequeña escala bajo condiciones locales que favorecen la acumulación de MOPF como la presencia de condiciones de velocidad heterogénea como las reportadas en este estudio para el tramo alto.

Por otro lado, los organismos que respiran por tegumento como Tanypodinae y Chironominae también se relacionaron con el tramo alto. El incremento de este rasgo en el tramo alto coincide con lo reportado por Statzner, Dolédec \& Hugueny, (2004) y Reynaga \& Dos Santos, (2012) acerca de la relación entre este tipo de respiración y la mayor captación de oxígeno en las partes altas de las quebradas por la reareación producida por la acción de la corriente. Por otro lado, Motta et al. (2017), reportan mayor abundancia de este rasgo en sitios con concentraciones bajas de oxígeno en un río tropical de la Isla de Providencia.

Así mismo, en los tramos altos en los muestreos de diciembre (E2) y febrero (E4), hubo mayor cantidad de organismos con tamaño pequeño $(5-10 \mathrm{~mm})$; se sugiere que puede estar relacionado con la alta velocidad del flujo, que se ve reflejada en una menor inversión en el crecimiento por parte de los organismos como estrategia para buscar refugios y evitar ser arrastrados por la corriente (Townsend \& Hildrew, 1994; Brooks et al., 2005; Beche, Mcelravy, \& Resh, 2006; Reynaga \& Dos Santos, 2012; Dolédec, Tilbian, \& Bonada, 2017).

Los tramos bajos en los muestreos de enero y febrero (E3 y E4) se asociaron con la presencia de organismos raspadores como NeOelmis sp. (larva) que se alimentan de micrófitas, respiración por branquias como Andesiops sp., y forma del cuerpo aplanada. La predominancia de organismos con estructuras especializadas como las branquias a menores altitudes en ríos de montaña coincide con lo reportado por Tomanova \& Usseglio-Polatera, (2007); Statzner \& Beche, (2010); Reynaga \& Dos Santos, (2012), acerca de una mayor tolerancia a menores valores de oxígeno con este tipo de respiración.

La expresión de los rasgos descrita, se refleja en los estimadores de diversidad funcional, especialmente en la riqueza (RF) y la divergencia (DF). Respecto a la mayor RF en el tramo alto, se interpreta como un espectro dimensional más amplio en la expresión de rasgos con menor grado de redundancia entre los grupos de macroinvertebrados (Villéger, et al., 2008; Stubbington, Bogan, Bonada, Boulton, Datry, Leigh \& Vander Vorste, 2017). Se destaca en el muestreo E3, una reducción en la RF en ambos tramos, lo que coincide con un periodo de menor velocidad de flujo, esto se explica en un mayor grado de redundancia en la expresión de los rasgos de los macroinvertebrados con la consecuente reducción en el espectro multidimensional funcional. La DF mayor en el tramo alto corresponde con la tendencia a una mayor dominancia de grupos como Orthocladiinae, Chironominae, Simulium sp. y Andesiops sp., que a su vez expresan rasgos relevantes y característicos en la comunidad (Villéger, et al., 2008; Menezes, Baird \& Soares, 2010).

En cuanto a las asociaciones entre las variables físicas y los rasgos funcionales, se encontró que valores altos de velocidad se relacionan con estrategia de movilidad de adherencia al sustrato, endobentónico y volador. Esto se explica, teniendo en cuenta que los organismos encontrados en las rocas en ríos de montaña tienen estructuras especializadas para adherirse al sustrato (uñas anales o tarsales y ventosas) que les confieren cierta resistencia contra la fuerza de arrastre (Townsend \& Hildrew, 1994; Lamouroux et al., 2004; Tomanova \& Usseglio-Polatera, 2007; Reynaga \& Dos Santos, 2012). Asimismo, los organismos 
endobentónicos pueden escapar de las altas velocidades, enterrándose en los sedimentos del lecho. Lo anterior corrobora la importancia de la velocidad en la expresión de estos rasgos de la comunidad de macroinvertebrados (Lamouroux et al., 2004; Tomanova et al., 2006; Tomanova \& Usseglio-Polatera, 2007; Príncipe et al., 2010; Dolédec et al., 2017).

Se concluye que se presentaron cambios entre tramos y muestreos en las condiciones hidráulicas y grupos funcionales de los macroinvertebrados. Los tramos altos se caracterizaron por: alta velocidad de flujo, mayor abundancia de taxones como Orthocladiinae, Andesiops sp., Simulium sp. y aumento en la ocurrencia de categorías de rasgos funcionales como gremio trófico MOPF (colectores), respiración por tegumento y tamaño del cuerpo pequeño. En contraste, los tramos bajos se asociaron con la presencia de organismos como Nectopsyche sp. y categorías de rasgos como raspadores que se alimentan de micrófitas, respiración por branquias y forma del cuerpo aplanada. Se resalta el papel de la velocidad del flujo como una variable determinante en la ocurrencia del rasgo funcional de tipo de movilidad específicamente de las categorías adherido al sustrato, endobentónico y volador. Asimismo, se resalta la diversidad funcional como una expresión de los rasgos descritos, por lo cual se relaciona en los tramos altos con la dominancia de taxones como Orthocladiinae, Chironominae, Simulium sp. y Andesiops sp.

Se recomienda para futuros trabajos, determinar la diversidad taxonómica y diversidad funcional en diferentes escalas espaciales evaluando diferentes variables físicas y químicas con el fin de aproximarse a una comprensión menos sesgada del funcionamiento de los ecosistemas; de igual manera evaluar los rasgos funcionales con un mayor número de muestreos en un período interanual al igual que incluir un mayor número de rasgos morfológicos, fisiológicos, ecológicos.

Declaración de ética: los autores declaran que están de acuerdo con esta publicación; que no existe conflicto de interés de ningún tipo; y que ha cumplido con todos los requisitos y procedimientos éticos y legales pertinentes. Todas las fuentes de financiamiento se detallan plena y claramente en la sección de agradecimientos. El respectivo documento legal firmado se encuentra en los archivos de la revista.

\section{AGRADECIMIENTOS}

Al grupo de Investigación Unidad de Ecología en Sistemas Acuáticos, UDESA de la UPTC por el apoyo y asesoría. Al macroproyecto "Modelación de idoneidad de hábitat de la comunidad y grupos funcionales de macroinvertebrados" financiado por Colciencias en la convocatoria No. 733 de 2015 y la Gobernación de Boyacá. Agradecemos a Gustavo Giraldo y César Tamaris por sus comentarios y aportes que contribuyeron al mejoramiento de la propuesta inicial.

\section{RESUMEN}

Introducción: La estructura, diversidad taxonómica y diversidad funcional del ensamblaje de macroinvertebrados acuáticos son estimadores sensibles a las variaciones del medio circundante. El ambiente físico es un factor fundamental, donde el sustrato y la dinámica hidráulica modifican la disponibilidad de recursos, la estructuración, el funcionamiento de la comunidad y del ecosistema. Objetivo: Determinar la estructura, diversidad taxonómica y diversidad funcional del ensamblaje de macroinvertebrados bajo diferentes condiciones abióticas en el río Hato Laguna, tributario del Lago de Tota. Métodos: Se recolectaron 120 muestras biológicas en cuatro momentos de muestreos en dos tramos en el río. Se midieron in situ profundidad, velocidad del flujo, ancho de canal y rugosidad del sustrato. Los rasgos funcionales evaluados fueron: longitud corporal, forma corporal, tipo de respiración, movilidad, gremio trófico y grupos funcionales alimenticios. Resultados: Se identificaron un total de 38 taxones; los órdenes Diptera, Coleoptera y Trichoptera presentaron la mayor riqueza de familias y géneros. Hubo mayor abundancia de Orthocladiinae, Andesiops sp. y Simulium sp. en condiciones de alta velocidad del flujo en los tramos altos; Nectopsyche sp. fue abundante en los tramos bajos relacionados con menores velocidades y mayor ancho del canal. Según los índices de diversidad taxonómica se presentó variación por tramos y muestreos sin observarse tendencias. En los tramos altos, el gremio trófico MOPF (colectores), respiración por tegumento y tamaño del cuerpo pequeño fueron característicos. En los tramos bajos, el ensamblaje fue de 
tipo raspador con respiración por branquias y forma del cuerpo aplanada. La riqueza, divergencia y equidad funcional fueron mayores en el tramo alto. En el Análisis de Correspondencia Canónica, los mayores valores de ancho y velocidad se relacionaron con categorías adherido al sustrato y endobentónico, mientras que valores bajos de ancho y rugosidad se relacionaron con las categorías de forma del cuerpo aplanado, recurso alimentario micrófitas y tamaño del cuerpo máximo. Conclusiones: El sistema presentó variación en las condiciones abióticas, en la estructura, diversidad taxonómica y funcional de los macroinvertebrados. Se resalta el papel de la velocidad del flujo como una variable determinante de la estructura y función del ensamblaje de macroinvertebrados. Asimismo, se destaca la diversidad funcional como una expresión de los rasgos descritos, por lo cual se relaciona en los tramos altos con la dominancia de taxones como Orthocladiinae, Chironominae, Simulium sp. y Andesiops sp.

Palabras clave: ríos andinos; insectos acuáticos; rasgos funcionales; variables físicas; hidráulica; Lago de Tota.

\section{REFERENCIAS}

Acuña, V., Muñoz, I., Giorgi, A., Omella, M., Sabater, F., \& Sabater, S. (2005). Drought and postdrought recovery cycles in an intermittent Mediterranean stream: structural and functional aspects. Journal of the North American Benthological Society, 24(4), 919-933.

Allan, J. D., \& Castillo, M. M. (2007). Stream ecology: structure and function of running waters. London, England: Chapman \& Hall.

Beche, L. A., Mcelravy, E. P., \& Resh, V. H. (2006). Long-term seasonal variation in the biological traits of benthic-macroinvertebrates in two Mediterraneanclimate streams in California, USA. Freshwater Biology, 51(1), 56-75.

Bonada, N., Prat, N., Resh, V. H., \& Statzner, B. (2006). Developments in aquatic insect biomonitoring: a comparative analysis of recent approaches. Annual Review of Entomology, 51, 495-523.

Bonada, N., Doledec, S., \& Statzner, B. (2007). Taxonomic and biological trait differences of stream macroinvertebrate communities between mediterranean and temperate regions: implications for future climatic scenarios. Global Change Biology, 13(8), 1658-1671.

Bonada, N., Rieradevall, M., \& Prat, N. (2007). Macroinvertebrate community structure and biological traits related to flow permanence in a Mediterranean river network. Hydrobiologia, 589(1), 91-106.

Brooks, A. J., Haeusler, T. I. M., Reinfelds, I., \& Williams, S. (2005). Hydraulic microhabitats and the distribution of macroinvertebrate assemblages in riffles. Freshwater Biology, 50(2), 331-344.
Carrera, P., \& Gunkel, G. (2003). Ecology of a high Andean stream, Rio Itambi, Otavalo, Ecuador. Limnologica, 33(1), 29-43.

Chao, A., \& Jost, L. (2012). Coverage-based rarefaction and extrapolation: standardizing samples by completeness rather than size. Ecology, 93(12), 2533-2547.

Chará-Serna, A. M., Chará, J. D., Zúñiga, M. C., Pedraza, G. X., \& Giraldo, L. P. (2010). Clasificación trófica de insectos acuáticos en ocho quebradas protegidas de la ecorregión cafetera colombiana. Universitas Scientiarum, 15(1), 27-36.

Chará-Serna, A. M., Chará, J. D., Zúñiga, M. del C., \& Pearson, R. G. (2012). Diets of leaf litter associated invertebrates in three tropical streams. Annales de Limnologie- International Journal of Limnology, 48 (2), 139-144.

Chevenet, F., Doléadec, S., \& Chessel, D. (1994). A fuzzy coding approach for the analysis of long-term ecological data. Freshwater biology, 31(3), 295-309.

Clarke, K. R. (1993). Non-parametric multivariate analyses of changes in community structure. Australian journal of ecology, 18(1), 117-143.

CONPES 3801 (2014). Manejo Ambiental Integral de la Cuenca del Lago de Tota. Consejo Nacional de Política Económica y Social-CONPES, Departamento Nacional de Planeación-DNP, Bogotá, D.C.

Death, R. G., \& Winterbourn, M. J. (1995). Diversity patterns in stream benthic invertebrate communities: the influence of habitat stability. Ecology, 76(5), 1446-1460.

Dolédec, S., Tilbian, J., \& Bonada, N. (2017). Temporal variability in taxonomic and trait compositions of invertebrate assemblages in two climatic regions with contrasting flow regimes. Science of the Total Environment, 599, 1912-1921.

Domínguez, E. \& Fernández, H. (2009). Macroinvertebrados bentónicos sudamericanos: sistemática y biología. Buenos Aires, Argentina: Fundación Miguel Lillo.

Elosegi, A. \& Sabater, S. (2009). Conceptos y técnicas en ecología fluvial. Bilbao, España: Fundación BBVA.

Encalada, A. C., Flecker, A.S., Poff, N.L., Suárez, E., Herrera-R, G. A., Ríos-Touma, B., \& Anderson, E.P. (2019). A global perspective on tropical montane rivers. Science, 365(6458), 1124-1129.

González, C \& Cañón, J (2016). Impactos de la variabilidad climática y las actividades humanas en la dinámica hidrológica del lago de Tota (Tesis de Maestría). Universidad de Antioquia.

Guisande, C., Barreiro, A., Maneiro, I., Riveiro, I., \& Vergara, A. (2006). Tratamiento de datos. España: Ed. Díaz de Santos. 
Guzmán-Soto C.J., \& Tamarís-Turizo, C.E. (2014). Hábitos alimentarios de individuos inmaduros de Ephemeroptera, Plecoptera y Trichoptera en la parte media de un río tropical de montaña. Revista de Biología Tropical, 62, 169-178.

Heino, J. (2005). Functional biodiversity of macroinvertebrate assemblages along major ecological gradients of boreal headwater streams. Freshwater Biology, 50(9), 1578-1587.

Henriques-Oliveira, A. L., Nessimian, J. L., \& Dorvillé, L. F. M. (2003). Feeding habits of chironomid larvae (Insecta: Diptera) from a stream in the Floresta da Tijuca, Rio de Janeiro, Brazil. Brazilian Journal of Biology, 63(2), 269-281.

Holzenthal, R. W., \& Rios-Touma, B. (2018). Nectopsyche of Ecuador: a new species from the high Andean páramo and redescription of Nectopsyche spiloma (Ross) (Trichoptera: Leptoceridae). PeerJ, 6, e4981.

IDEAM. (2018). Banco de Datos del Instituto de Hidrología, Meteorología y Estudios Ambientales. Valores mensuales de precipitación Río Hato Laguna. Recuperado de: http: //www.ideam.gov.co., accedida en noviembre de 2018.

Jacobsen, D. (2004). Contrasting patterns in local and zonal family richness of stream invertebrates along an Andean altitudinal gradient. Freshwater Biology, 49(10), 1293-1305.

Lamouroux, N., Dolédec, S., \& Gayraud, S. (2004). Biological traits of stream macroinvertebrate communities: effects of microhabitat, reach, and basin filters. Journal of the North American Benthological Society, 23(3), 449-466.

Laliberté, E., Legendre, P., Shipley, B., Laliberté, M. E. (2014). Package ' $F D$ '. Measuring functional diversity from multiple traits, and other tools for functional ecology. R package version 1.2.

Lepš, J., \& Šmilauer, P. (2003). Multivariate analysis of ecological data using CANOCO. New York, USA: Cambridge University Press.

Lewis, W. M. (2008). Physical and chemical features of tropical flowing waters. En Tropical stream ecology (pp. 1-21). Academic Press.

Longo, M., Zamora, H., Guisande, C., \& Ramírez, J. J. (2010). Dinámica de la comunidad de macroinvertebrados en la quebrada Potrerillos (Colombia) respuesta a los cambios estacionales de caudal. Limnetica, 29(2), 195-210.

Longo, M. \& Blanco, J.F. (2014). Shredders are abundant and species-rich in tropical continental-island loworder streams: Gorgona Island, Tropical Eastern Pacific, Colombia. Revista de Biología Tropical 62: 85-105.
Lujan, N. K., Roach, K. A., Jacobsen, D., Winemiller, K. O., Vargas, V. M., Ching, V. R., \& Maestre, J. A. (2013). Aquatic community structure across an Andes-to-Amazon fluvial gradient. Journal of Biogeography, 40(9), 1715-1728.

Matthaei, C. D., Arbuckle, C. J., \& Townsend, C. R. (2000). Stable surface stones as refugia for invertebrates during disturbance in a New Zealand stream. Journal of the North American Benthological Society, 19(1), 82-93.

Menezes, S., Baird, D. J., \& Soares, A. M. (2010). Beyond taxonomy: a review of macroinvertebrate trait-based community descriptors as tools for freshwater biomonitoring. Journal of Applied Ecology, 47(4), 711-719.

Merrit, R., Cummins, K., \& Berg, M. (2008). An Introduction to the Aquatic Insects of North America. Dubuque: Kendall/ Hunt Publishing.

Mesa, L. M. (2010). Hydraulic parameters and longitudinal distribution of macroinvertebrates in a subtropical andean basin. Interciencia, 35(10), 759-764.

Meza-S, A. M., Rubio-M, J., Dias, G. L., \& Walteros, J.M. (2012). Calidad de agua y composición de macroinvertebrados acuáticos en la Subcuenca Alta del Río Chinchiná, Colombia /Water quality and composition of aquatic macroinvertebrates in the subwatershed of river Chinchiná, Colombia. Caldasia, 34 (2), 443-456.

Montgomery, D. R., \& Buffington, J. M. (1998). Channel processes, classification, and response. In R. Naiman, R. E. Bilby., S. Kantor (Eds.), River ecology and management: lessons from the Pacific Coastal Ecoregion (pp. 13-42). New York, USA: Springer-Verlag.

Moreno, C. E. (2001). Manual de métodos para medir la biodiversidad. Universidad Veracruzana.

Moreno González, H.F (2015). Análisis multiespectral mediante imágenes Landsat para identificación de zonas degradadas en el área circundante en la Laguna de Tota, departamento de Boyacá. (Tesis de especialización). Universidad Militar Nueva Granada. Bogotá.

Moretti, M.S. (2005). Decomposição de detritos foliares e sua colonização por invertebrados aquáticos em dois córregos na Cadeia do Espinhaço (MG). (Tesis de Maestría). Instituto de Ciências Biológicas, Belo Horizonte, Brasil.

Motta, A.J., Longo, M. L. M., \& Aranguren-Riaño, N. (2017). Variación temporal de la diversidad taxonómica y rasgos funcionales de los macroinvertebrados acuáticos en ríos temporales en la isla de Providencia, Colombia. Actualidades Biológicas, 39(107), 82-100.

Motta, A.J., Obregón, N \& Vimos, L.D. (2018). Modelación de idoneidad de hábitat de la comunidad y grupos funcionales de macroinvertebrados. (Tesis 
de Maestría, Maestría en Hidrosistemas, Facultad de Ingeniería, Pontificia Universidad Javeriana).

Moya, C., Valdovinos, C., Moraga, A., Romero, F., Debels, P., \& Oyanedel, A. (2009). Patrones de distribución espacial de ensambles de macroinvertebrados bentónicos de un sistema fluvial Andino Patagónico. Revista chilena de historia natural, 82(3), 425-442.

Muñoz, I., Romaní, A., Rodrigues, A., González, J., \& García, E. (2009). Relaciones tróficas en el ecosistema fluvial. En A. Elosegi \& S. Sabater (Eds.), Conceptos y técnicas en ecología fluvial (pp. 344-351). Bilbao, España. Fundación BBVA.

Oyanedel, A., Valdovinos, C., Azócar, M., Moya, C., Mancilla, G., Pedreros, P., \& Figueroa, R. (2008). Patrones de distribución espacial de los macroinvertebrados bentónicos de la cuenca del río Aysén (Patagonia chilena). Gayana (Concepción), 72(2), 241-257.

Parasiewicz, P. (2007). The MesoHABSIM model revisited. River Research and Applications, 23(8), 893-903.

Pavitra, S.P., Low, V.L., Tan, T.K., Lim, Y.A., Ya'cob, Z. (2019). Temporal variation in diversity and community structure of preimaginal black-flies (Diptera: Simuliidae) in a tropical forest reserve in Malaysia. Acta Tropica, 202, 105275.

Pennak, R. W. (1989). Fresh invertebrates of the United States; Protozoa to Mollusca. USA: Johan Wily and Sons.

Petchey, O. L., Hector, A., \& Gaston, K. J. (2004). How do different measures of functional diversity perform? Ecology, 85(3), 847-857.

Plazas Figueroa, D.A (2016). Diseño de medidas de manejo ambiental orientadas a la disminución de los niveles de eutrofización; estudio de caso en la microcuenca del río Hatolaguna en el Humedal Lago de Tota (Municipios de Aquitania-Sogamoso, Boyacá).

Poff, N. L. (1997). Landscape filters and species traits: toward mechanistic understanding and prediction in stream ecology. Journal of North American Benthological Society, 16 (2):391-409.

Prat, N., Ribera, C., Rieradevall, M., Villamarin, C., \& Acosta, R. (2013). Distribution, abundance and molecular analysis of genus Barbadocladius Cranston \& Krosch (Diptera, Chironomidae) in tropical, high altitude Andean streams and rivers. Neotropical entomology, 42(6), 607-617.

Príncipe, R. E., Gualdoni, C. M., Oberto, A. M., Raffaini, G. B., \& Corigliano, M. C. (2010). Spatial-temporal patterns of functional feeding groups in mountain streams of Córdoba, Argentina. Ecología Austral, 20, 257-268

R Development Core Team. (2019). R: A language and environment for statistical computing. Vienna,
Austria: R Foundation for Statistical Computing; 2011. URL: https://www. R-project.org.

Ramírez, A., \& Gutiérrez-Fonseca, P. E. (2014). Estudios sobre macroinvertebrados acuáticos en América Latina: avances recientes y direcciones futuras. Revista de Biología Tropical, 62, 9-20.

Rempel, L., Richardson, J., Healey, M. (2000). Macroinvertebrate community structure along gradients of hydraulic and sedimentary conditions in a large gravel-bed river. Freshwater Biology, 45(1). 57-73

Reynaga, M. C., \& Dos Santos, D. A. (2012). Rasgos biológicos de macroinvertebrados de ríos subtropicales: patrones de variación a lo largo de gradientes ambientales espacio-temporales. Ecología Austral, $22(2), 112-120$

Richards, C., Haro, R., Johnson, L., \& Host, G. (1997). Catchment and reach-scale properties as indicators of macroinvertebrate species traits. Freshwater Biology, 37(1), 219-230.

Ricotta, C. (2005). A note on functional diversity measures. Basic and Applied Ecology, 6(5), 479-486.

Ríos-Touma, B., Encalada, A. C., \& Prat Fornells, N. (2011). Macroinvertebrate assemblages of an Andean high-altitude tropical stream: The importance of season and flow. International Review of Hydrobiology, 96(6), 667-685.

Rivera, C., Zapata, A., Pérez, D., Morales, Y., Ovalle, H., \& Alvarez, J. P. (2010). Caracterización limnológica de humedales de la planicie de inundación del río Orinoco (Orinoquía, Colombia). Acta Biológica Colombiana, 15(1), 145-166.

Rodríguez-Barrios, J., Ospina-Tórres, R., \& Turizo-Correa, R. (2011). Grupos funcionales alimentarios de macroinvertebrados acuáticos en el río Gaira, Colombia. Revista de Biología Tropical, 59(4), 1537-1552.

Roldán, G.; Zúñiga, M.; Zamora, L.; Reinoso, G.; Longo, M.(2014). Colombia. En: Alonso-Eguía Lis, P.; Mora, J.; Campbell, B.; Springer, M. Diversidad, conservación y uso de los macroinvertebrados dulceacuícolas de México, Centroamérica, Colombia, Cuba y Puerto Rico (63-93). Jiutepec, México: Instituto Mexicano de Tecnología del Agua.

Roleček, J., Chytrý, M., Hájek, M., Lvončík, S., \& Tichý, L. (2007). Sampling design in large-scale vegetation studies: Do not sacrifice ecological thinking to statistical purism! Folia Geobotanica, 42(2), 199.

Rolls, R. J., Leigh, C., \& Sheldon, F. (2012). Mechanistic effects of low-flow hydrology on riverine ecosystems: Ecological principles and consequences of alteration. Freshwater Science, 31(4), 1163-1186.

Schmera, D., Heino, J., Podani, J., Erős, T., \& Dolédec, S. (2017). Functional diversity: a review of methodology 
and current knowledge in freshwater macroinvertebrate research. Hydrobiologia, 787(1), 27-44.

Statzner, B., Dolédec, S., \& Hugueny, B. (2004). Biological trait composition of European stream invertebrate communities: assessing the effects of various trait filter types. Ecography, 27(4), 470-488.

Statzner, B., \& Beche, L. A. (2010). Can biological invertebrate traits resolve effects of multiple stressors on running water ecosystems? Freshwater Biology, $55,80-119$

Stubbington, R., Bogan, M. T., Bonada, N., Boulton, A. J., Datry, T., Leigh, C., \& Vander Vorste, R. (2017). The biota of intermittent rivers and ephemeral streams: aquatic invertebrates. In Intermittent rivers and ephemeral streams (pp. 217-243). Academic Press.

Tachet, H., Richoux, P., Bournaud, M., \& Usseglio-Polatera, P. (2002). Invertébrés d'eau douce. París, Francia: CNRS Éditions.

Tilman, D., 2001. Functional diversity. In: Levin, S.A. (Ed.), Encyclopedia of Biodiversity, vol. 3. Academic Press, NewYork, pp. 109-120.

Tomanova, S., Goitia, E., \& Helešic, J. (2006). Trophic levels and functional feeding groups of macroinvertebrates in neotropical streams. Hydrobiologia, 556 (1), 251-264

Tomanova, S., \& Usseglio-Polatera, P. (2007). Patterns of benthic community traits in neotropical streams: relationship to mesoscale spatial variability. Fundamental and Applied Limnology/Archiv für Hydrobiologie, 70(3), 243-255.

Townsend, C. R., \& Hildrew, A. G. (1994). Species traits in relation to a habitat templet for river systems. Freshwater Biology, 31(3), 265-275.

Vannote, R. L., Minshall, G. W., Cummins, K. W., Sedell, J. R., \& Cushing, C. E. (1980). The river continuum concept. Canadian journal of fisheries and aquatic sciences, 37(1), 130-137.

Vásquez-Ramos, J.; Reinoso, G. (2012). Estructura de la fauna béntica en corrientes de los Andes colombianos. Revista Colombiana de Entomología. 38(2), 351-358.
Villada, S., Triana, L., \& Dias, L. (2017). Grupos funcionales alimentarios de insectos acuáticos en quebradas andinas afectadas por agricultura y minería. Caldasia, 39(2), 370- 387.

Villamarín, C. (2008). Estructura y composición de las comunidades de macroinvertebrados acuáticos en ríos altoandinos del Ecuador y Perú. Diseño de un sistema de medida de la calidad del agua con índices multimétricos (Doctoral dissertation, Tesis Doctoral, Departamento de Ecología, Facultad de Biología, Universidad de Barcelona).

Villéger, S., Mason, N. W., Mouillot, D. (2008). New multidimensional functional diversity indices for a multifaceted framework in functional ecology. Ecology, 89 (8): 2290-2301.

Vimos-Lojano, D. V. (2017). Influencia de las condiciones hidráulicas e hidrológicas en la variación espacial y temporal de las comunidades de macroinvertebrados acuáticos en ríos altoandinos de cabecera al sur de Ecuador (Tesis Doctoral). Universitat Politecnica de Valencia, Valencia, España.

Vimos-Lojano, D. J., Martínez-Capel, F., \& Hampel, H. (2017). Riparian and microhabitat factors determine the structure of the EPT community in Andean headwater rivers of Ecuador. Ecohydrology, 10(8), e1894.

Vimos-Lojano, D., Martínez-Capel, F., Hampel, H., \& Vázquez, R. (2019). Hydrological influences on aquatic communities at the mesohabitat scale in high Andean streams of southern Ecuador. Ecohydrology, 12(1), e2033.

Violle C., Navas ML., Vile D., Kazakou E., Fortune C., Hummel I., \& Garnier E. (2007). Let the concept of trait be functional! Oikos, $116(5), 882-892$.

Wadeson, R. A. (1994). A geomorphological approach to the identification and classification of instream flow environments. Southern African Journal of Aquatic Science, 20(1-2), 38-61.

Wanumen, A.M (2019). Dinámica de la cobertura del suelo y percepción del recurso hídrico en la cuenca del lago de Tota. (Tesis de Maestría). Universidad Distrital Francisco José de Caldas. Bogotá. 